Draft VERSion OCTOBER 23, 2018

Typeset using LATEX RNAAS style in AASTeX62

\title{
A CATAlog of Stars observed Simultaneously by KePler AND TESS
}

\author{
Thomas Barclay ${ }^{1,2}$ and Geert Barentsen ${ }^{3,4}$ \\ ${ }^{1}$ NASA Goddard Space Flight Center, 8800 Greenbelt Rd, Greenbelt, MD 20771 \\ ${ }^{2}$ University of Maryland, Baltimore County, 1000 Hilltop Circle, Baltimore, MD 21250, USA \\ ${ }^{3}$ NASA Ames Research Center, Moffett Field, CA 94035 \\ ${ }^{4}$ Bay Area Environmental Research Institute, P.O. Box 25, Moffett Field, CA 94035, USA
}

Keywords: surveys, catalogs

The Kepler spacecraft provided the first long-baseline, high-precision photometry for large numbers of stars (Borucki et al. 2010). This enabled the discovery of thousands of new exoplanets (e.g. Rowe et al. 2014; Morton et al. 2016; Thompson et al. 2018; Mayo et al. 2018), and the characterization of myriad astrophysical phenomena (e.g. Bedding et al. 2011; Thompson et al. 2012; Garnavich et al. 2016). However, one of the challenges with interpreting Kepler data has been that no instrument has provided a comparison dataset. Therefore, the replication of Kepler time-series data has remained elusive. While there have been efforts to reproduce observations of Kepler's transiting planets using the Hubble and Spitzer Space Telescopes, this has been limited to a small number of stars and short observing baselines (Beichman et al. 2016).

The Transiting Exoplanet Survey Satellite (TESS) launched in April 2018 and began science operations in July 2018 (Ricker et al. 2015). During the TESS primary mission, it will survey $85 \%$ of the sky. NASA's first two observatories dedicated to discovering exoplanets, Kepler and TESS, were simultaneously operating during 2018. While the K2 mission surveyed the ecliptic plane, TESS targets fields outside the ecliptic. However, during September 2018, a small region of the sky was observed simultaneously by both TESS and Kepler as part of TESS's Sector 2 (Aug 22 - Sep 20, 2018) and Kepler's K2 Campaign 19 (Sep 7 - Sep 26, 2018). The overlap region was $0.5 \mathrm{deg}^{2}$.

TESS full-frame image mode collected the entire region, but K2 targets must be pre-selected. We used the Web TESS Target Tool (Mukai \& Barclay 2017), available at the TESS Science Support Center website, to determine which K2 Campaign 19 targets $^{1}$ were observed by TESS. We identified 171 K2 targets that fell inside TESS Sector 2 field of view, all of which are observed at 30-minute cadence by both missions. We cross-matched these 171 targets to the TESS Input Catalog (Stassun et al. 2018) and found 169 matches within 6". The two sources with no match were fainter than $\mathrm{Kp}=18$. A portion of the catalog is shown in Table 1.

The targets range in brightness in the TESS bandpass (Tmag) from 6.7-18.4 ( $K p=7.7-19.2)$, with 93 targets brighter than $T m a g=15$, and 17 brighter than $T m a g=12$. The majority of the sample are main-sequence stars. We cross-matched the sample with Gaia and found 153 matches (Gaia Collaboration et al. 2016, 2018). Using the Gaia distance estimates from Bailer-Jones et al. (2018), we found targets range in distance from 80-6500 pc, with a mean distance of 800 pc. The brightest target is the multiple star system HD $218928(K p=7.7)$. The sample also contains approximately 30 galaxies which were observed for the K2 Supernova Experiment. There is a Seyfert galaxy (EPIC 245934248), a source with high-proper motion (EPIC 245924999, 180 milliarcsec/yr), a white dwarf (EPIC 251456407), and an RR Lyra star candidate (EPIC 251813843). Of these 171 targets, 81 were previously observed during K2's Campaign 12 (Dec 2016 - March 2017).

This dataset provides the first simultaneous, long-duration, high-precision observations of the same targets from different space-based observatories and presents an excellent opportunity to explore instrumental systematics present in the two telescopes. For Kepler, we can look to see the extent to which the optics, detectors, or electronics are contributing signals to the data, and disentangle these from astrophysical contributions. For TESS we can use Kepler data, with its considerably higher photometric precision, as a ground-truth.

1 The K2 target lists are available at https://keplerscience.arc.nasa.gov. 
Table 1. A sample of the targets observed simultaneously by Kepler and TESS

\begin{tabular}{|c|c|c|c|c|c|c|c|c|c|c|}
\hline EPIC ID & TICID & Gaia DR2 ID & $\mathrm{RA}$ & Dec & $K p$ & Tmag & $G$ & $\begin{array}{l}\text { Teff } \\
(\mathrm{K})\end{array}$ & $\begin{array}{l}\text { Radius } \\
\qquad\left(R_{\odot}\right)\end{array}$ & $\begin{array}{l}\text { Dist } \\
\text { (pc) }\end{array}$ \\
\hline 245925582 & 4554210 & 2413051302797084416 & 347.998206 & -11.933554 & 7.66 & 6.68 & 7.49 & 5047 & 9.55 & 253 \\
\hline 245929348 & 4579916 & 2413014469157323264 & 348.407601 & -11.749342 & 9.32 & 8.10 & 9.02 & 4130 & 23.55 & 702 \\
\hline 245931711 & 4582469 & 2413392224416454528 & 348.516775 & -11.629405 & 9.94 & 9.41 & 9.79 & 6296 & 1.18 & 148 \\
\hline 245927251 & 4520668 & 2413067451874115840 & 347.867863 & -11.852871 & 10.93 & 10.51 & 10.83 & 6736 & 1.85 & 429 \\
\hline
\end{tabular}

Note-Table 1 is published in its entirety as a comma-separated values file. A portion is shown here for guidance regarding its form and content. Tmag is from the TESS Input Catalog (Stassun et al. 2018), RA, Dec and Kp are from the K2 Ecliptic Plane Input Catalog (Huber et al. 2016), G, Teff and radius are from Gaia DR2 (Gaia Collaboration et al. 2016, 2018), and distance is inferred from Gaia DR2 (Bailer-Jones et al. 2018). This catalog is also available from Figshare (Barclay \& Barentsen 2018).

We thank the PIs of K2 programs with targets in our catalog: Matthew Burleigh, William Cochran, Courtney Dressing, Ryan Foley, Joyce Guzik, J. J. Hermes, Andrew Howard, Daniel Huber, Adam Jensen, Marshall Johnson, Armin Rest, Robert Szabo, Dennis Stello, and Thomas Barclay. This work has made use of data from the European Space Agency (ESA) mission Gaia (https://www.cosmos.esa.int/gaia), processed by the Gaia Data Processing and Analysis Consortium (DPAC, https://www.cosmos.esa.int/web/gaia/dpac/consortium). Funding for the DPAC has been provided by national institutions, in particular the institutions participating in the Gaia Multilateral Agreement.

Software: IPython (Perez \& Granger 2007), Jupyter (Kluyver et al. 2016), Pandas (McKinney 2010), Astropy (Astropy Collaboration et al. 2013), Astroquery (Ginsburg et al. 2016), TopCAT (Taylor 2005), K2fov (Mullally et al. 2016), tvguide (Mukai \& Barclay 2017) 


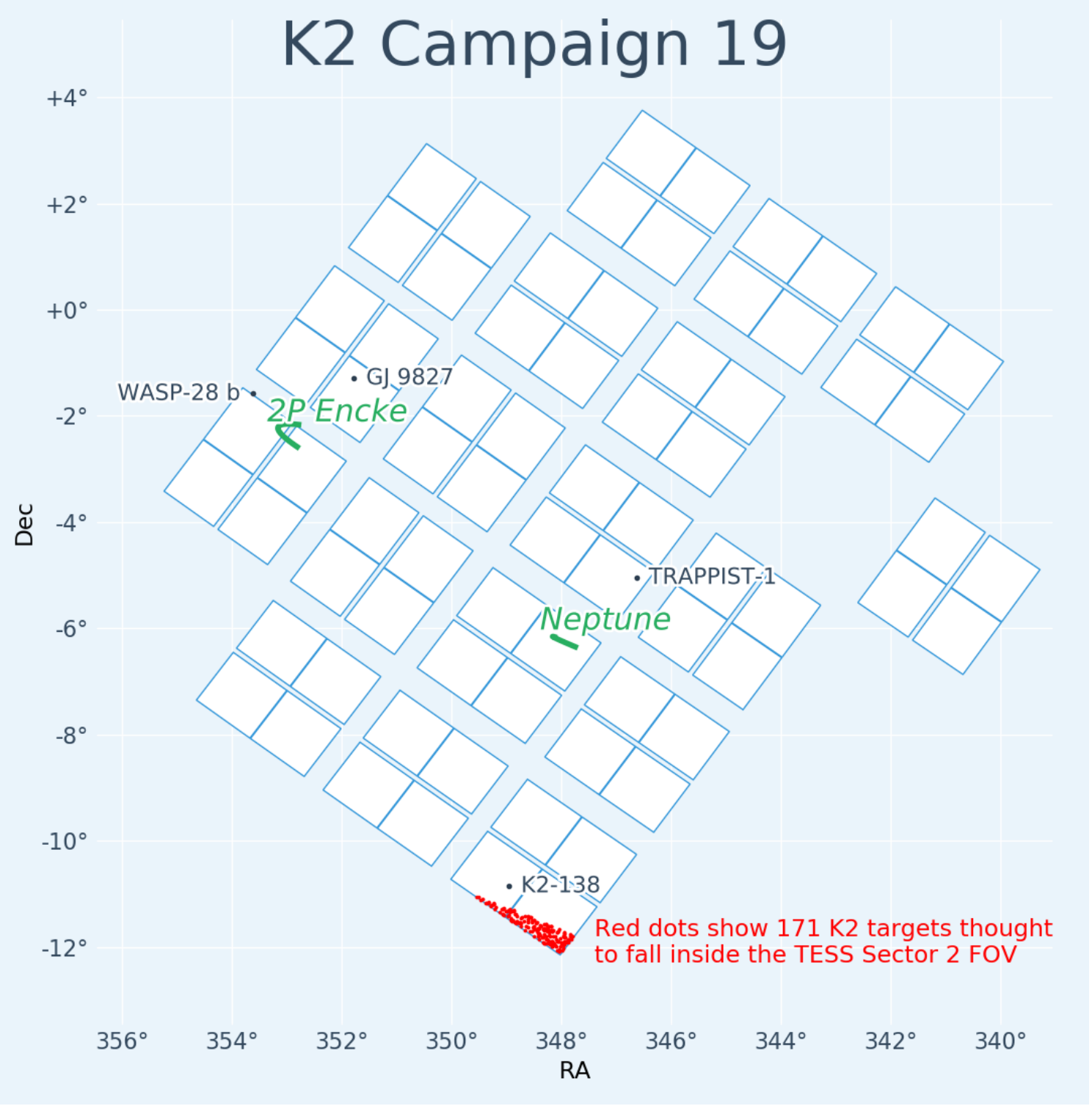

Figure 1. The location of K2 Campaign 19. In red are the 171 targets that overlap with the field of view of TESS Sector 2 . This figure is not included in the RNAAS published version of this paper.

\section{REFERENCES}

Astropy Collaboration, Robitaille, T. P., Tollerud, E. J., et al. 2013, A\&A, 558, A33

Bailer-Jones, C. A. L., Rybizki, J., Fouesneau, M., Mantelet, G., \& Andrae, R. 2018, AJ, 156, 58

Barclay, T., \& Barentsen, G. 2018, A catalog of stars observed simultaneously by Kepler and TESS, Figshare, doi:10.6084/m9.figshare.7227260.v1.

https://doi.org/10.6084/m9.figshare.7227260

Bedding, T. R., Mosser, B., Huber, D., et al. 2011, Nature, 471,608

Beichman, C., Livingston, J., Werner, M., et al. 2016, ApJ, 822,39
Borucki, W. J., Koch, D., Basri, G., et al. 2010, Science, 327,977

Gaia Collaboration, Prusti, T., de Bruijne, J. H. J., et al. 2016, A\&A, 595, A1

Gaia Collaboration, Brown, A. G. A., Vallenari, A., et al. 2018, A\&A, 616, A1

Garnavich, P. M., Tucker, B. E., Rest, A., et al. 2016, ApJ, 820,23

Ginsburg, A., Parikh, M., Woillez, J., et al. 2016, astroquery v0.3.1, Zenodo, doi:10.5281/zenodo.44961. https://doi.org/10.5281/zenodo.44961 
Huber, D., Bryson, S. T., Haas, M. R., et al. 2016, ApJS, 224,2

Kluyver, T., Ragan-Kelley, B., Pérez, F., et al. 2016, in Positioning and Power in Academic Publishing: Players, Agents and Agendas, ed. F. Loizides \& B. Scmidt (IOS Press), 87-90. https://eprints.soton.ac.uk/403913/

Mayo, A. W., Vanderburg, A., Latham, D. W., et al. 2018, AJ, 155, 136

McKinney, W. 2010, in Proceedings of the 9th Python in Science Conference, ed. S. van der Walt \& J. Millman, 51 $-56$

Morton, T. D., Bryson, S. T., Coughlin, J. L., et al. 2016, ApJ, 822, 86

Mukai, K., \& Barclay, T. 2017, tvguide: A tool for determining whether stars and galaxies are observable by TESS., v1.0.1, Zenodo, doi:10.5281/zenodo.823357. https://doi.org/10.5281/zenodo.823357

Mullally, F., Barclay, T., \& Barentsen, G. 2016, K2fov: Field of view software for NASA's K2 mission, Astrophysics Source Code Library, , , ascl:1601.009
Perez, F., \& Granger, B. E. 2007, Computing in Science Engineering, 9, 21

Ricker, G. R., Winn, J. N., Vanderspek, R., et al. 2015, Journal of Astronomical Telescopes, Instruments, and Systems, 1, 014003

Rowe, J. F., Bryson, S. T., Marcy, G. W., et al. 2014, ApJ, 784,45

Stassun, K. G., Oelkers, R. J., Pepper, J., et al. 2018, AJ, 156, 102

Taylor, M. B. 2005, in Astronomical Society of the Pacific Conference Series, Vol. 347, Astronomical Data Analysis Software and Systems XIV, ed. P. Shopbell, M. Britton, \& R. Ebert, 29

Thompson, S. E., Everett, M., Mullally, F., et al. 2012, ApJ, 753, 86

Thompson, S. E., Coughlin, J. L., Hoffman, K., et al. 2018, ApJS, 235, 38 